\title{
Effect of cypermethrin on the postnatal development of the medulla oblongata and the possible protective role of melatonin in albino rats
}

\author{
Marwa A. Al-Gholam, Noha M. Issa \\ Department of Anatomy and Embryology, Faculty of Medicine, Menoufia University, Shebin El-Kom, Egypt
}

\begin{abstract}
Previous studies have shown that cypermethrin (CYP), a broad spectrum pesticide has a teratogenic effect on rat offspring born to an exposed dam with no information on its effect on the development of the brain. To the best of our knowledge, this research is the first attempt to study the postnatal development medulla oblongata of rat offspring exposed to CYP during the perinatal period and the possible neuroprotective role of melatonin. The offspring of treated female rats were organized into control, melatonin (1 mg/kg/day orally); CYP (12 mg/kg/day orally); and CYP/melatonin groups. The mothers received treatments from day 6 of gestation until day 21 after birth. At Postnatal days 7 and 21, the animals were sacrificed and their medulla oblongata was removed and subjected to histological, immunohistochemical, and electron microscopic studies. CYP induced neuronal degeneration by chromatolysis and pyknosis. Nuclear changes, cytoplasmic vacuolation, damage mitochondria, and breakdown of RER were also detected. Reduction of microtubule-associated protein-2 (MAP2), myelin basic protein (MBP), and oligodendrocyte transcription factor expressions and increment of glial fibrillary acidic protein expression in the medulla oblongata of the developing rats were observed. On the other hand, melatonin led to an obvious improvement of the injured medulla oblongata tissues and ameliorating the damaging effects of CYP. In conclusion, melatonin has protected rats against CYP-induced histopathological and immunohistochemical changes. This may be due to the protection of MAP-2, conservation of MBP, an increment of oligodendrocytes, and alleviation of astrogliosis.
\end{abstract}

Key words: Cypermethrin, Melatonin, Medulla oblongata, Immunohistochemistry

Received July 23, 2020; 1st Revised September 1, 2020; 2nd Revised September 15, 2020; Accepted September 16, 2020

\section{Introduction}

Cypermethrin (CYP), type II synthetic pyrethroid, is a broad-spectrum pesticide. It plays a role in agriculture, pro-

\footnotetext{
Corresponding author:

Marwa A. Al-Gholam (iD

Department of Anatomy and Embryology, Faculty of Medicine, Menoufia University, Shebin El Kom, 32511, Egypt

E-mail: marwaelgholam@yahoo.com, marwa.mohyeldeen@med. menofia.edu.eg
}

tection of foodstuff, disease vector control, and home pest control [1]. CYP is widely used as an insecticide in developing countries to control many species of insects. Humans are exposed to CYP mainly during the application or from pyrethroids residues such as those detected in vegetables, fruits, cow's milk, and bread [2]. CYP is a hydrophobic molecule that can easily pass through the cell membrane, disturbing its structure and cause leakage of cytoplasmic enzymes [3]. CYP produces reactive oxygen species (ROS) and causes direct damage to DNA proportional to the dose [4]. There is a direct reaction between ROS and cellular biomolecules leading to damage of lipids, proteins, and DNA in cells caus- 
ing cell death [5]. An imbalance between the amount of free radicals generated and antioxidant defenses in the body is one of the reasons for CYP toxicity [1].

Melatonin is a neuroendocrine substance produced by the pineal gland and proposed as a neuroprotective agent based on its ability to function as a free radical scavenger [6]. Several studies have reported that melatonin can attenuate mitochondrial damage and neuroinflammation and induce proliferation [7]. Long-term administration of melatonin would lower the post-lesion region-specific histological consequences of Temporal lobe epilepsy in the rostrocaudal axis of the dorsal hippocampus [8].

Previous reports showed that fetuses are very sensitive to chemical toxicants, particularly during critical periods of development [9]. Although considered to be safe for household applications, some studies indicated the adverse effects of cypermethrin on the brain of laboratory animals [10]. A recent study reported a toxic effect of cypermethrin on the sensory retina of the offspring their mothers exposed to it during the prenatal period [11].

The aim of the present study, therefore, was to assess the neurotoxic effect of cypermethrin on the postnatal development of rat medulla oblongata and the possible neuroprotective role of melatonin.

\section{Materials and Methods}

\section{Chemicals and drugs}

Commercial Cypermethrin: It is an oily solution containing $250 \mathrm{~g} / \mathrm{l}$ of the active ingredient cypermethrin, which belongs to type-II pyrethroids "cyano pyrethroids" and has the chemical name: [a-Cyano-(3-phenoxyphenyl) methyl ( \pm )cis/trans-3-(2,2-dichlorovinyl)2,2-dimethyl-cyclopropanecarboxylate], and molecular formula $\mathrm{C} 22 \mathrm{H} 19 \mathrm{O} 3 \mathrm{NCl} 2$ [12]. It was diluted with distilled water to concentration levels convenient to be used throughout the whole experiment, in which $1 \mathrm{ml}$ of diluted working solution contained $12 \mathrm{mg}$ of cypermethrin.

Melatonin powder (Bio Basic Canada, Inc., Markham, ON, Canada): $10 \mathrm{mg}$ of the powder was dissolved in $1 \mathrm{ml}$ of absolute ethanol because of its instability in non-sterile solutions then mixed with tap water to a concentration of $1 \mathrm{mg} /$ $\mathrm{ml}$. The solution of melatonin was freshly prepared [13].

\section{Animals}

Thirty sexually mature female albino rats and ten male albino rats (for mating) of Sprague-Dawley strain, weighted between 200-250 g were allowed for laboratory rat chow diet and water ad-libitum, and the study was approved by the faculty of medicine, Menoufia University institutional ethical committee on the animal experiment. Each three of them were housed overnight with a sexually mature male albino rat for mating, and every morning vaginal smears were taken and microscopically examined for the presence of sperms. The presence of sperms in the smears marked the occurrence of mating and was considered as the 1st day of gestation then the male offspring of these mothers were divided into the following groups:

\section{Experimental design}

Control group: consisted of ten male offspring their mothers were fed ad libitum and allowed free water supply.

Melatonin treated group: consisted of ten male offspring of the mothers that received melatonin $1 \mathrm{mg} / \mathrm{kg} /$ day orally by gavage [13].

Cypermethrin treated group (CYP): consisted of ten male offspring their mothers received $12 \mathrm{mg} / \mathrm{kg} /$ day of cypermethrin by oral gavage [14].

Cypermethrin and melatonin treated group (CYP/melatonin): consisted of ten male offspring their mothers received $12 \mathrm{mg} / \mathrm{kg} /$ day of cypermethrin [14] and melatonin $1 \mathrm{mg} / \mathrm{kg} /$ day orally by gavage [13].

The treatment was conducted from the 6th day of pregnancy until postnatal day 21. Five rats from each group were sacrificed at the age of postnatal day (PND) 7 and five at the age of PND 21.

\section{Histopathological examination}

At the end of the experiment, the rats were anesthetized by diethyl ether inhalation. We prepared serial sections of 5 $\mu \mathrm{m}$ thickness along the sagittal plane. The sections in the lateral $1.9-2.4 \mathrm{~mm}$ of the rat medulla oblongata were collected for histological and immunohistochemical studies according to Luo et al. [15]. Sections of Medulla oblongata were fixed in $10 \%$ buffered formalin ( $\mathrm{pH} 7.4$ ) for 48 hours.

The tissues were dehydrated in ascending ethyl alcohol followed by two changes of xylene. The tissues were impregnated in paraffin wax and then embedded in paraffin wax. Sections were cut, dewaxed, hydrated, and stained with hematoxylin and eosin (H\&E) stain [16]. 


\section{Ultrastructure examination}

Small parts of the medulla oblongata $(1 \mathrm{~mm} \times 1 \mathrm{~mm} \times 2 \mathrm{~mm})$ at the lateral 1.9-2.4 $\mathrm{mm}$ level from the center [15] were taken and immediately immersed in buffered formol glutaraldehyde $(\mathrm{pH}=7.3)$. After an hour, the medulla oblongata segments were cut into about $1 \mathrm{~mm}^{3}$ specimens and fixed in buffered formol glutaraldehyde, $\mathrm{pH}$ 7.3, for 24 hours at $4^{\circ} \mathrm{C}$ and routinely osmicated in $1 \%$ osmium tetroxide. After dehydration with graded ethanol series, the tissues were embedded in Araldite (Huntsman Advanced Materials, Salt Lake City, UT, USA). Then, TB stain was used to stain the semithin sections as a preliminary step for the selection of the areas needed for the ultrathin examination. Ultrathin sections were stained with lead citrate and uranyl acetate and were viewed under a transmission electron microscope.

\section{Immunohistochemical examination}

For the immunohistochemical study, the $5-\mu \mathrm{m}$ medulla oblongata paraffin sections were deparaffinized and rehydrated in descending grades of alcohol. Following the blocking of endogenous peroxidase activity with $3 \% \mathrm{H}_{2} \mathrm{O}_{2}$ in methanol and nonspecific binding sites with a protein blocker, the primary antibody anti Mab2 (Mouse monoclonal, 1:500, Abcam, Cambridge, UK), anti-myelin basic protein (MBP; Rabbit polyclonal, 1:300, Abcam), anti-oligodendrocyte transcription factor (anti-olig2; Rabbit monoclonal, 1:100; Abcam) and anti-GFAP (Rabbit polyclonal, 1:300, LabVision, Fermont, CA, USA) added with overnight incubation in a cold room. On day 2, the biotinylated goat-polyvalent secondary antibody (Vector, Peterborough, UK) was added at a concentration of $2 \%$ for 30 minutes $\left(37^{\circ} \mathrm{C}\right)$ followed by the addition of the avidin-biotin-peroxidase complex (Vector). All steps were performed at room temperature in a humidity chamber.

\section{Morphometric and statistical analysis}

For immunohistochemical quantitative assessment of MAP-2, MBP, and GFAP area percentage and the number of Olig2+ve cells, ImageJ $1.47 \mathrm{v}$ software (National Institutes of Health, USA) was used. A minimum of three sections per animal was assessed. Five nonoverlapping fields per section were randomly taken. The values calculated were utilized for comparison and statistical analyses.

The results are expressed as the mean \pm standard deviation. The data were analyzed using a one-way analysis of variance (ANOVA) followed by a post hoc Bonferroni test. A
$P<0.05$ was considered statistically significant.

\section{Results}

There was no significant difference in the all examined parameters between the control and Melatonin treated groups.

\section{Histopathological findings}

\section{hematoxylin and eosin staining}

H\&E sections of the medulla oblongata of the control group revealed numerous well-differentiated motor neurons that appear varied in shape with rounded nuclei. A large number of small capillaries and different forms of neuroglia could be detected. At the age of PND 21, the neurons appeared larger with an increment of the number of oligodendrocytes (Fig. 1).

The medulla oblongata of offspring their mothers exposed to cypermethrin showed a neuronal loss, degenerated neurons, and chromatolysis at the age of 7 days. At the age of 21 day, the above changes were severed with a delay of the cytogenesis (Fig. 1).

Histomorphology of cypermethrin and melatonin treated rat (CYP/melatonin) medulla oblongata at the age of 7 and 21 days postnatal was represented with clear architectural details such as a motor neuron, centrally placed nucleus within the neuron, and myelinated nerve fibers (Fig. 1).

\section{Semithin sections finding}

Examination of semithin sections on day 7 postnatal demonstrated normal neurons with vesicular nuclei and prominent nucleoli scattered in a background formed of neuroglia cells. Different types of neuroglia can be identified. Astrocyte has a pale nucleus and cytoplasm; the oligodendrocytes are small, rounded, and darkly stained. The microglia are easy to be distinguished from oligodendrocytes because of their irregular shape. On day 21, semithin sections showed large neurons, numerous microglia, and more myelinated fibers (Fig. 2). The medulla oblongata of offspring their mothers exposed to cypermethrin semithin sections showed an increased number of astrocytes and the section is devoid of myelinated axons (Fig. 2). At the age of 21 days these changes were exaggerated (Fig. 2). Cypermethrin and melatonin treated rats (CYP/melatonin), the medulla oblongata on day 7 , and 21 postnatal showed more or less normal architecture 

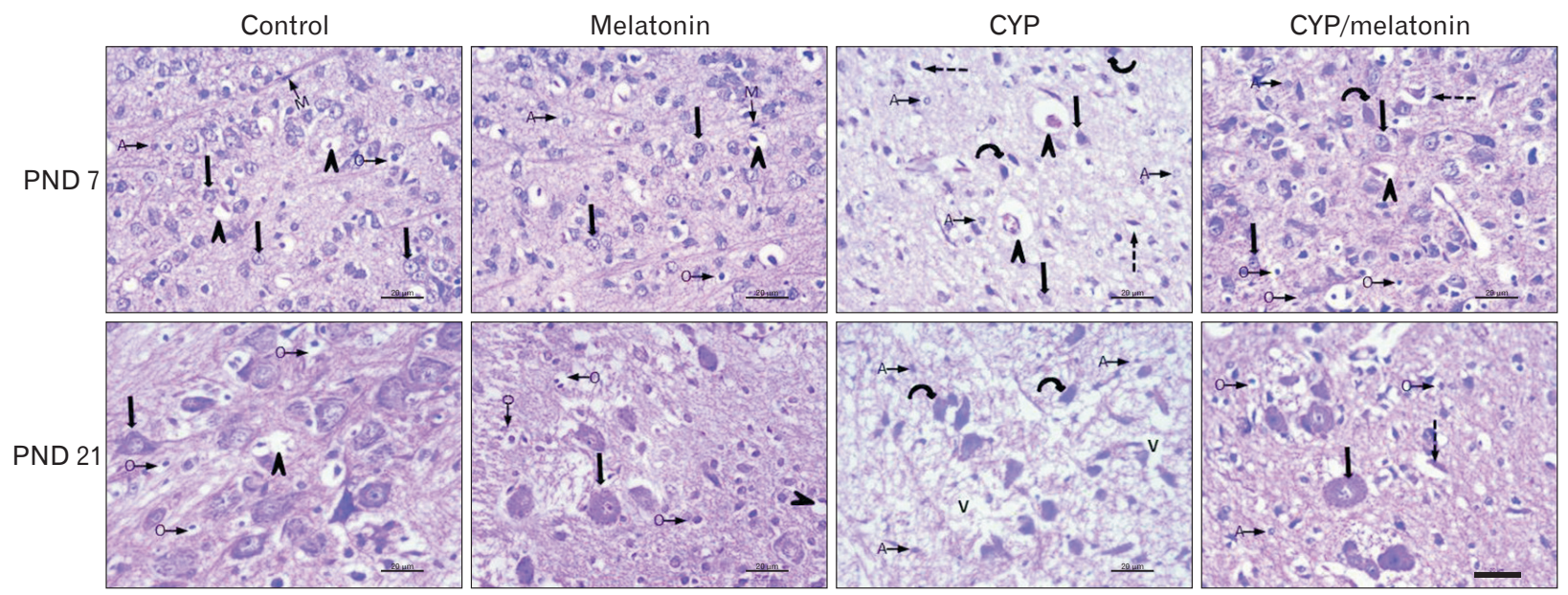

Fig. 1. Representative H\&E staining of rat medulla oblongata of control and melatonin aged 7 days: showing medulla neurons appeared small in size, varied in shape and had round nuclei (black arrows). The neuropil contains different neuroglia (O, A, and M) and blood vessels (arrowheads). Medulla oblongata of CYP treated group of the same age shows neurons with central chromatolysis (curved arrows), degenerated neurons and pyknotic nuclei (dashed arrows), dilated blood vessels (black arrowheads) and numerous A. CYP/melatonin treated group resulted in almost normal appearing neuronal cells, except for chromatolysis (curved arrow) and degenerated neurons and pyknotic nuclei (dashed arrow) in some neurons. Increased number of $\mathrm{O}$ and deceased of $\mathrm{A}$. Control and melatonin groups aged 21 days, the neurons appear larger (black arrow). Many oligodendrocytes (arrows with rounded end) are observed in the neuropil. CYP treated group show chromatolysis of almost all neurons (curved arrows), a large number of A and v. Co-administration of CYP/melatonin shows more or less normal neurons (black arrow). However, some degenerated cells (curved arrow) with loss of nuclear details could be detected. Few A and more O could be observed. A, astrocyte; CYP, cypermethrin; M, microglia; O, oligodendrocyte; PND, postnatal day; v, vacuolated neuropil. Scale bar=20 $\mu \mathrm{m}$.
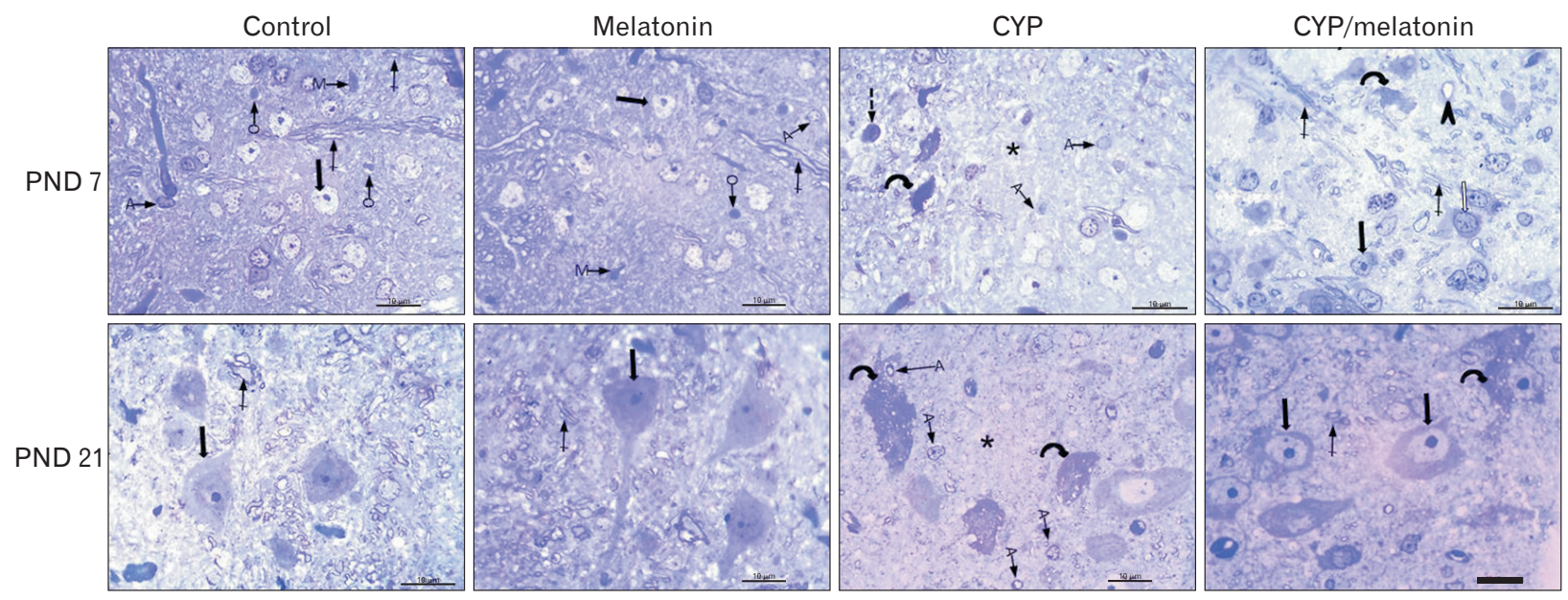

Fig. 2. Representative semi-thin blue-stained sections of the rat medulla oblongata of control and melatonin aged 7 days: showing normal neurons with vesicular nuclei and prominent nucleoli (black arrow), different neuroglia (A, O, and $\mathrm{M}$ ) and myelinated axons (crossed arrows). CYP treated group of the same age showing some shrunken neuronal bodies with an irregular outline, hardly identified nuclei, and deeply stained vacuolated cytoplasm (dashed arrow). Some neurons appear to have deeply stained nuclei and cytoplasm (curved arrow)., Numerous fine vacuoles were detected in neurons, numerous A, and neuropil devoid of myelinated axons (star). CYP/melatonin treated group shows nearly normal neurons with prominent nucleoli (black arrow), neurons containing coarse clumps of heterochromatin (white arrow), myelinated axons (crossed arrow). Darkly stained neurons (curved arrow) and dilated blood vessels are still observed. Sections of the control and melatonin groups aged 21 days showing large multipolar neurons with large nucleus \& prominent nucleolus (black arrow). The neuron is surrounded by dense neuropil containing more myelinated axons (crossed arrow). CYP treated group of the same age shows neurons with numerous fine vacuoles (curved arrows), a large number of A, and neuropil devoid of myelinated axons (star). CYP/melatonin treated group shows neurons with pale vesicular nuclei with prominent nucleoli (black arrows) and myelinated axons in the surrounding neuropil. However, few darkly stained vacuolated neurons are detected. A, astrocyte; CYP, cypermethrin; M, microglia; O, oligodendrocyte; PND, postnatal day. Scale bar=10 $\mu \mathrm{m}$. 
(Fig. 2).

\section{Ultrastructure finding}

The light microscopic results of our study were confirmed by ultrastructural examination. By electron microscopy, the neuronal cells on day 7 postnatal were characterized by vesicular nuclei with prominent and surrounded by regular nuclear. Their homogenous cytoplasm contains mitochondria and rough endoplasmic reticulum. Few numbers of myelinated axons with smooth regular contours can be detected (Fig. 3). On day 21, examination showed neurons with a large nucleus, the chromatin inside the nucleus was euchromatin mainly with less amount of heterochromatin. The cytoplasm of the neuron contained large mitochondria, free ribosomes, and Golgi apparatus. The neuron was surrounded by many myelinated axons (Fig. 3)

The medulla oblongata of offspring their mothers exposed to cypermethrin at the age of 7 and 21 days. The ultrastructural examination revealed few myelinated nerve fibers, large irregularly outlined nuclei, and irregularly distributed organelles in the cytoplasm. Numerous dilated channels of the rough endoplasmic reticulum and mitochondria were also seen. The nucleus has an irregular indented outline (Fig. 3).

Cypermethrin and melatonin treated rats (CYP/melatonin), ultrastructural observations exhibit a euchromatic nucleus, finely dispersed chromatin, and prominent nucleolus. More or less normal rER and myelinated axons could be detected. However, vacuolations at the age of 7 days and mild irregular nucleus and ballooned mitochondria at the age of 21 days were still detected (Fig. 3).
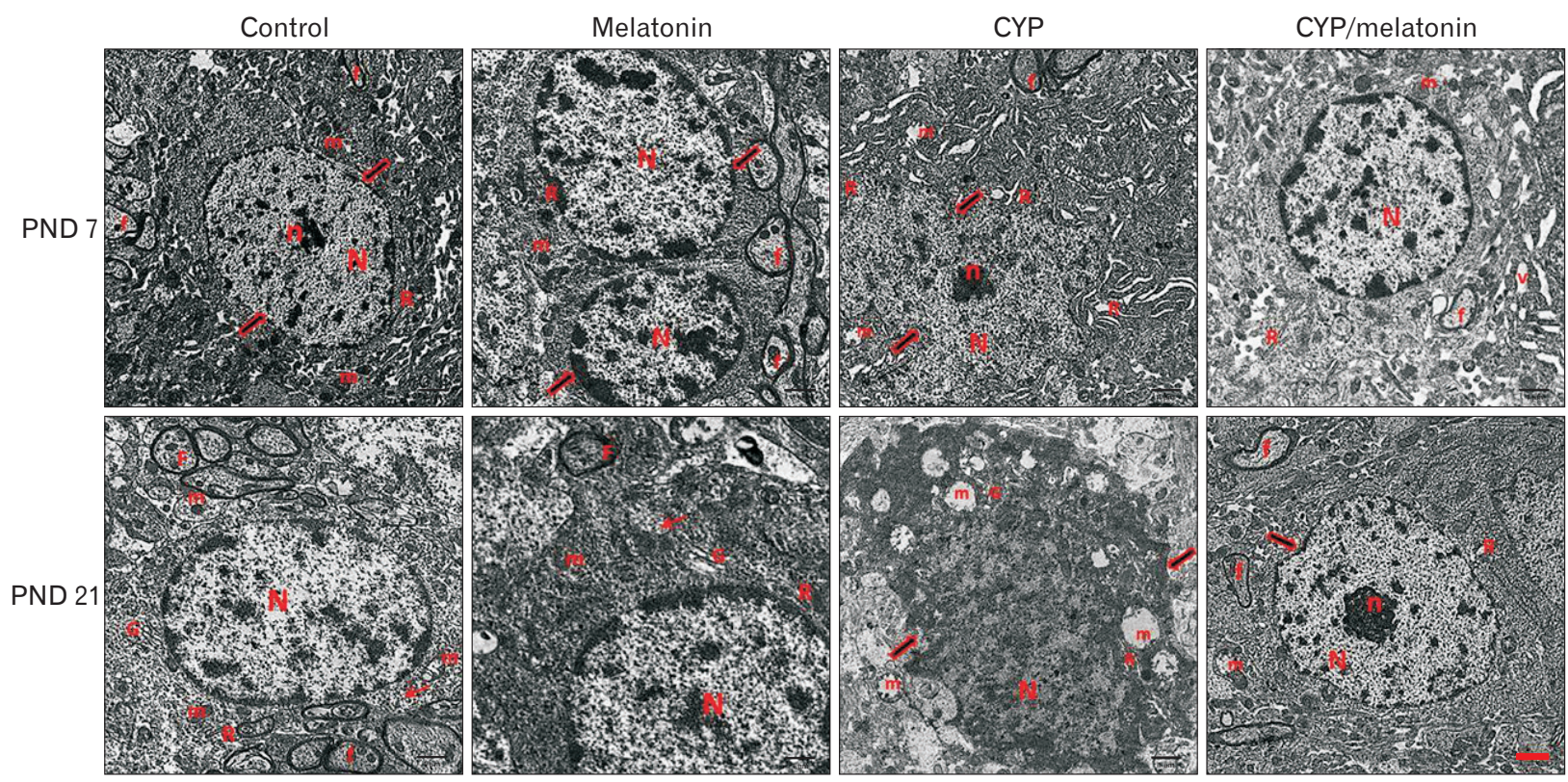

Fig. 3. An electron micrograph of a section in control and melatonin rat medulla oblongata aged 7 days shows motor neurons having rounded euchromatic $\mathrm{N}$ with regular nuclear membranes (arrow) and prominent $\mathrm{Nu}$. The homogenous cytoplasm contains normal $\mathrm{m}$ and rough endoplasmic R. Myelinated $\mathrm{f}$ with preserved myelin sheath are also observed. CYP treated group of the same age displays an irregular N surrounded by an indented nuclear membrane (arrows). The cytoplasm contains dilated cisternae of $\mathrm{rER}(\mathrm{R})$ and dilated $\mathrm{m}$ with destructed cristae $(\mathrm{m})$. Few myelinated $\mathrm{f}$ could be detected. CYP/melatonin treated group shows an apparently normal nerve cell with a regular $\mathrm{N}$ but the $\mathrm{N}$ still shows heterochromatin. The cytoplasm contains normal more or less normal RER (R) and $\mathrm{m}$. However, a small area of V could be still detected in the cytoplasm. A section of the control and melatonin group aged 21 days shows a large neuron with a large $\mathrm{N}$, the chromatin inside the $\mathrm{N}$ is euchromatin mainly and less amount of heterochromatin. The cytoplasm of the neuron contains large $\mathrm{m}$, G, and multiple free ribosomes (arrow). The neuron is surrounded by many myelinated axons (f). CYP treated group of the same age displays markedly affected neurons. The $\mathrm{N}$ is shrunken, darkly electron-dense, and irregular in shape with indentation of its nuclear membrane (arrows). Many dispersed irregularly shaped chromatin aggregates are demonstrated throughout the $\mathrm{N}$. The cell has an irregular outline with a shrinkage cytoplasm containing multiple ballooned $\mathrm{m}$ with disrupted cristae and dilated rER (R) and G. Medulla oblongata sections of CYP/melatonin treated group of the same age exhibit a euchromatic $\mathrm{N}$, fine dispersed chromatin, and prominent $\mathrm{n}$. More or less normal rER (R) and myelinated axons (f) could be detected. However, mild irregular $\mathrm{N}$ (arrow) and ballooned $\mathrm{m}$ are still detected (transmission electron microscope, $\times 17,500, \mathrm{Scale}$ bar $=1 \mu \mathrm{m}$ ). CYP, cypermethrin; f, fibers; G, Golgi apparatus; m, mitochondria; N, nucleus; n, nucleolus; Nu, nucleoli; PND, postnatal day; R, reticulum; V, vacuolation. 


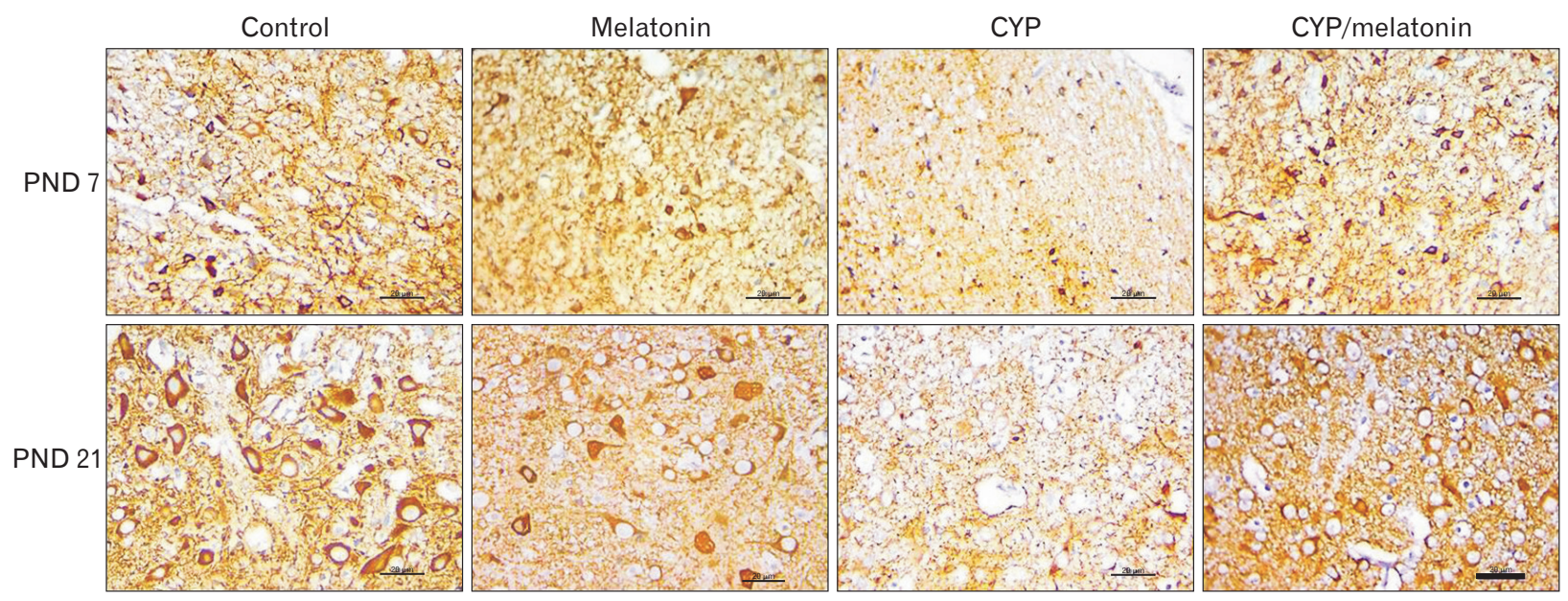

Fig. 4. Expression of MAP-2 in the medulla oblongata of the control, melatonin, CYP, CYP/melatonin groups at 7 and 21 PNDs. CYP administration dramatically decreased the expression of MAP-2 in rat medulla oblongata compared to the control group. Melatonin cotreatment significantly upregulated the expression of MAP-2. CYP, cypermethrin; MAP-2, microtubule-associated protein-2; PND, postnatal day. Scale bar $=20 \mu \mathrm{m}$.

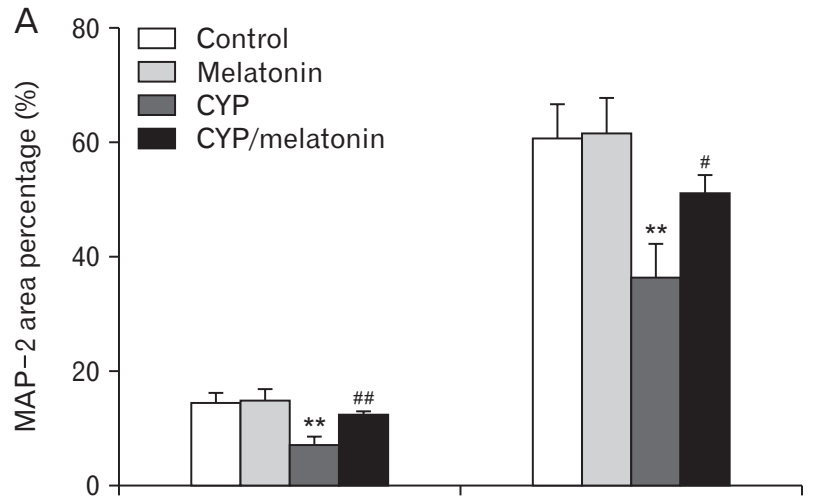

PND 7

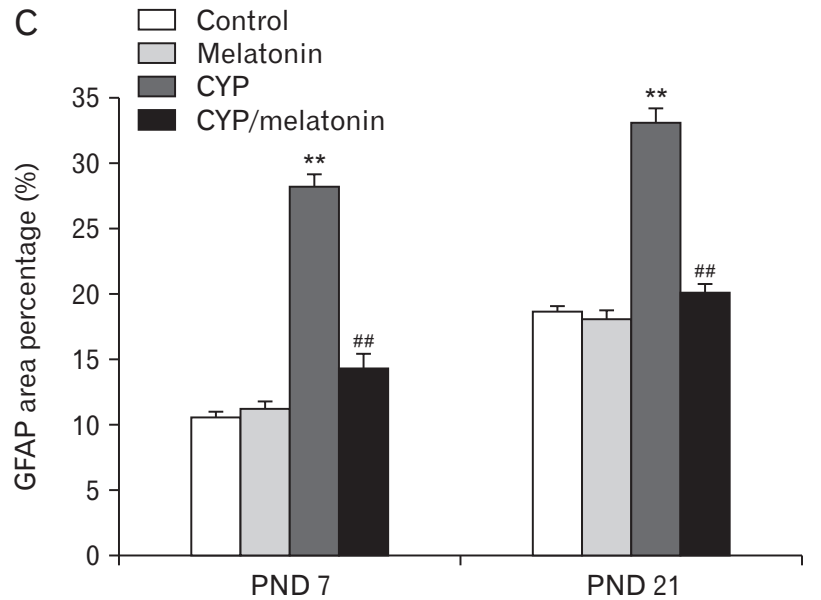

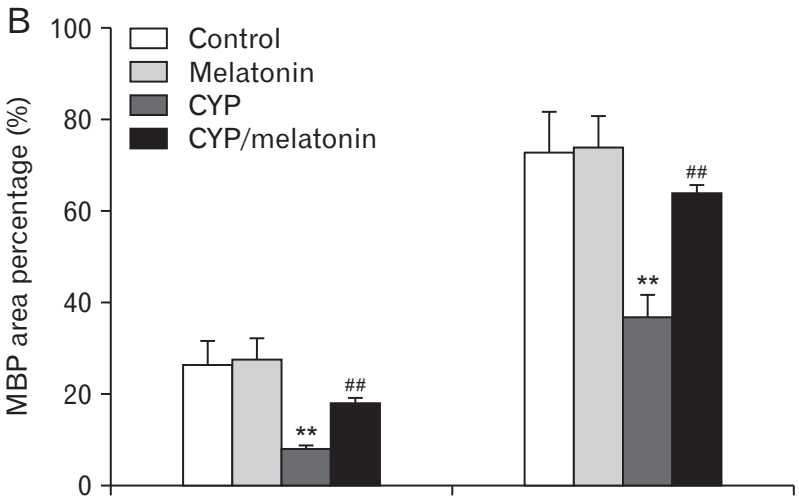

PND 7

PND 21

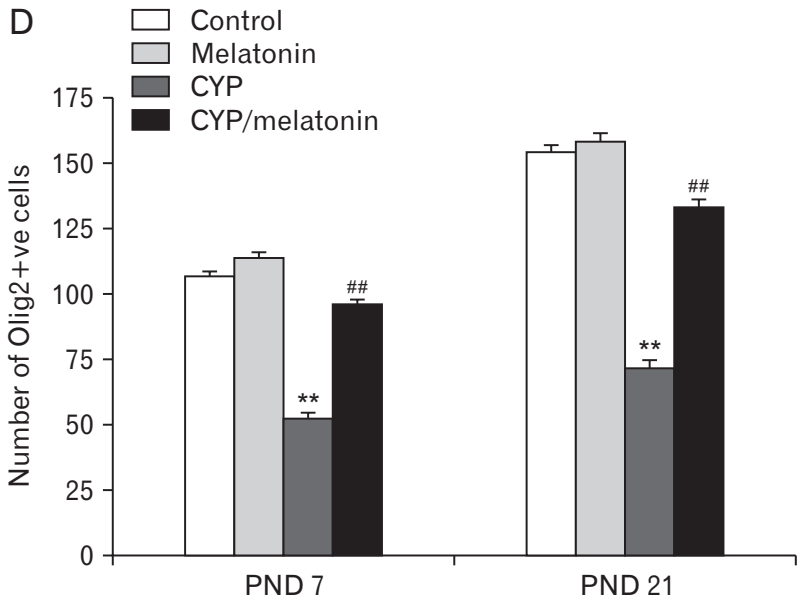

Fig. 5. Area percentage of (A) MAP-2, (B) MBP and (C) GFAP and (D) Olig2+ve cells in the medulla oblongata of the control, melatonin, CYP, CYP/melatonin groups at 7 and 21 PNDs. CYP, cypermethrin; GFAP, glial fibrillary acidic protein; MAP-2, microtubule-associated protein-2; MBP, myelin basic protein; Olig2, oligodendrocyte transcription factor; PND, postnatal day. ${ }^{* *} P<0.001$, compared with the control group; and ${ }^{\#} P<0.05$ and $^{\# \#} P<0.001$, compared with the CYP group. data are expressed as means \pm standard deviation. 


\section{Immunohistochemical staining}

MAP-2 expression, a protein that improves the stability of the dendritic cytoskeleton was increased in the developing rat medulla oblongata during the first and third postnatal weeks. The medulla oblongata of offspring prenatally exposed to cypermethrin showed significant downregulation (7.26 \pm 0.84 vs. $14.32 \pm 1.55$ and $36.36 \pm 6.00$ vs. $60.52 \pm 6.28$, $P<0.001)$ in MAP-2 expression during the first and third weeks postnatal respectively as compared to control group of the same age. Concomitant administration of melatonin with cypermethrin showed significant up-regulation $(12.3 \pm 1.03$ vs. $7.26 \pm 0.84$ and $50.9 \pm 7.95$ vs. $36.36 \pm 6.00, P<0.001$ and $P<0.05)$ of MAP-2 expression with age as compared to the cypermethrin group at the same age (Figs. 4, 5).

In the developing rat medulla oblongata during the first and third postnatal weeks, there is a substantial increase in MBP immunostaining (myelin sheath warping axon and oligodendroglia). Compared with the control group, Cypermethrin administration showed a steady significant decreased in MBP expression during the first and the third week postnatally ( $7.7 \pm 0.74$ vs. $26.22 \pm 4.92$ and $36.76 \pm 4.71$ vs. $72.32 \pm 8.91$ respectively, $P<0.001)$. Compared to the Cypermethrin
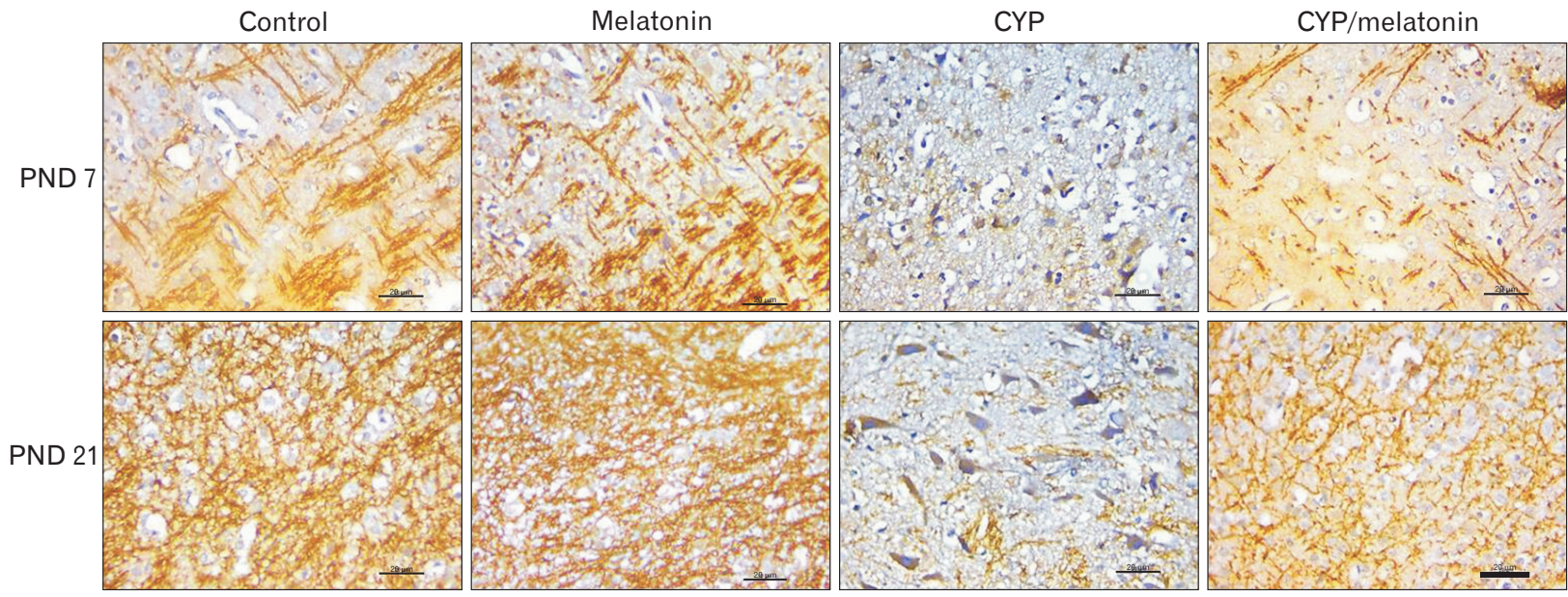

Fig. 6. Expression of MBP in the medulla oblongata of the control, melatonin, CYP, CYP/melatonin groups at 7 and 21 PNDs. CYP administration dramatically downregulated the expression of MBP in rat medulla oblongata compared to the control group. Melatonin cotreatment significantly increased the expression of MBP. CYP, cypermethrin; MBP, myelin basic protein; PND, postnatal day. Scale bar=20 $\mu$ m.
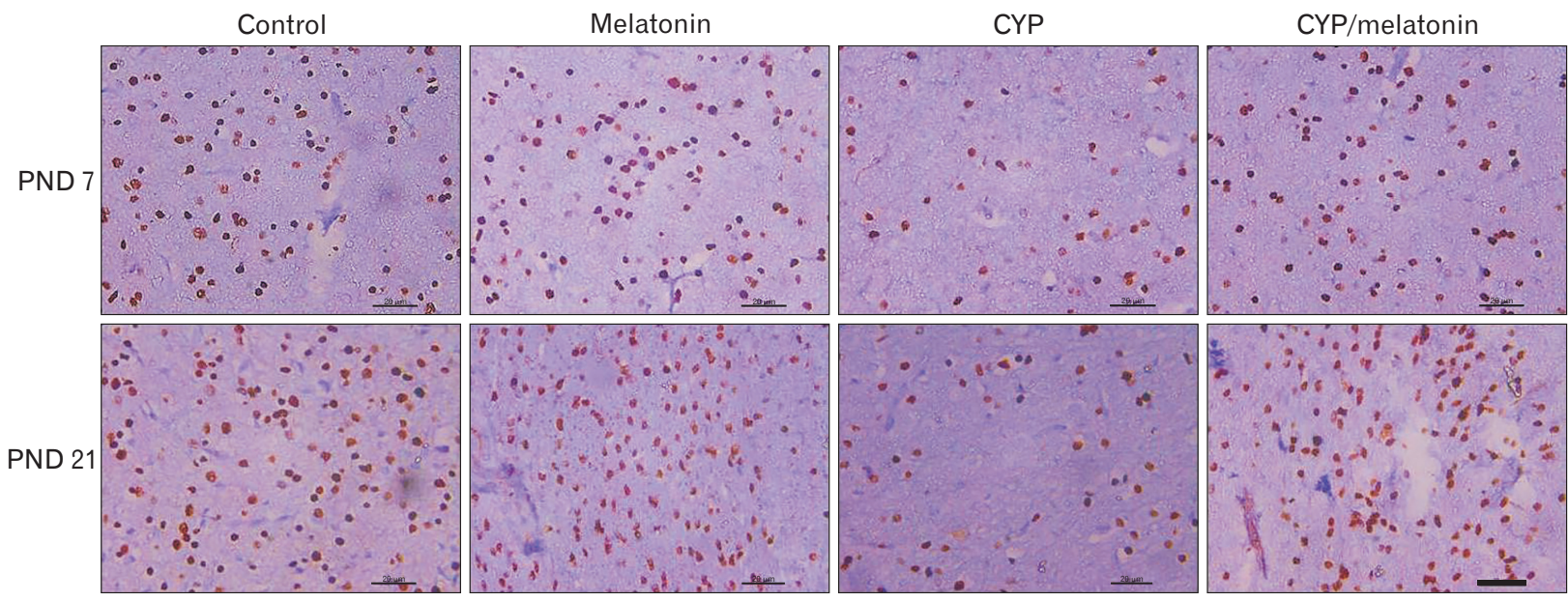

Fig. 7. Expression of Olig2 in the medulla oblongata of the control, melatonin, CYP, CYP/melatonin groups at 7 and 21 PNDs. CYP administration showed a reduction in the expression of Olig2+ve cells in rat medulla oblongata compared to the control group. Melatonin cotreatment increased the expression of Olig2+ve cells. CYP, cypermethrin; Olig2, oligodendrocyte transcription factor; PND, postnatal day. Scale bar $=20 \mu \mathrm{m}$. 

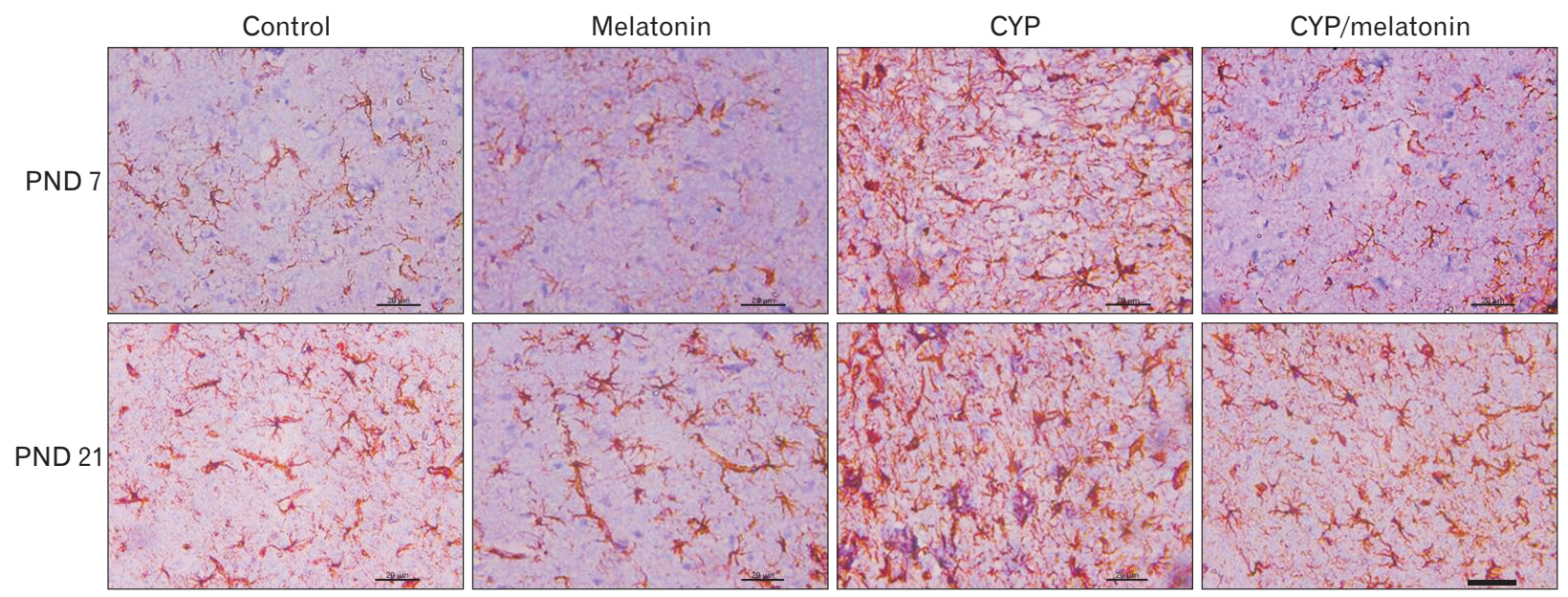

Fig. 8. Expression of GFAP in the medulla oblongata of the control, melatonin, CYP, CYP/melatonin groups at 7 and 21 PNDs. The administration of CYP showed an increase in the expression of GFAP in rat medulla oblongata compared to the control group. Melatonin treatment with CYP reduced the expression of GFAP. CYP, cypermethrin; GFAP, glial fibrillary acidic protein; PND, postnatal day. Scale bar $=20 \mu \mathrm{m}$.

group, at the same age, co-administration of melatonin with cypermethrin significantly increased expression (17.9 \pm 1.91 vs. $7.7 \pm 0.74$ and $63.14 \pm 4.99$ vs. $36.76 \pm 4.71, P<0.001)$ of $\mathrm{MBP}$ at PND 7 and 21 respectively (Figs. 5, 6).

Olig2, a marker of normal oligodendrocytes, the expression of it increased from the age of PND 7 to PND 21. Cypermethrin administration showed a significant decrease in Olig2 expression during the first and the third week postnatally $(52.4 \pm 3.85$ vs. $106.4 \pm 4.72$ and $71.6 \pm 5.27$ vs. $154 \pm 5.52$ respectively, $P<0.001)$ as compared to control. Administration of melatonin with cypermethrin significantly increased expression of Olig2 (95.8 \pm 3.7 vs. $52.4 \pm 3.85$ and $132.4 \pm 7.4$ vs. $71.6 \pm 5.27, P<0.001)$ at PND 7 and 21 respectively compared to CYP group (Figs. 5, 7).

GFAP immunostaining was conducted in the experimental groups for detection of the astroglial response. There was adramatic increase in GFAP expression in the Cypermethrin-treated group compared with the control group $(28.29 \pm 1.38$ vs. $10.55 \pm 1.09$ and $32.79 \pm 2.42$ vs. $18.6 \pm 0.94$, $P<0.001)$ at PND 7 and 21 respectively. This increment was significantly down-regulated in the cypermethrin and melatonin treated group $(14.34 \pm 2.17$ and $20.14 \pm 1.47, P<0.001)$ at PND 7 and 21 respectively (Figs. 5, 8).

\section{Discussion}

The brain being a coordinating and regulating system for body parts is a vital part of the organism. So, any damage as a result of physical, physiological, and chemical stress may have a serious impact on the entire organism [17]. Cypermethrin is a neurotoxin that can cross the blood-placental and brain barriers on prenatal exposure [18]. The cypermethrin residue may contaminate (food and water) causing chronic exposure which may endanger pregnant mothers and increasing the risk of development of CNS anomalies $[17,19]$. So, we need a low molecular substance that can cross those barriers and preserve the normal development of CNS [20]. Consequently, this study aimed to evaluate the role of melatonin in the preservation of normal medulla oblongata development of rats exposed prenatally to cypermethrin. This work showed neuronal degeneration and chromatolysis, reduction of myelinated fibers in the neuropil, and decrease number of oligodendrocytes in addition to increased astrocytes in the medulla oblongata of offspring their mothers exposed to cypermethrin on PND7. These changes were more severe on PND 21. This agrees with Grewal et al. [21]. Sayim et al. [10] explained this neurotoxic effect by an increase of $\mathrm{ChE}$ activity and the impairment of neural conductivity in the central and peripheral nervous systems. CYP administration led to ultrastructural features of apoptotic cell death in the form of nuclear indentation, chromatin margination, dilated rER cisternae, and abnormal mitochondria at PND7, in addition to cytoplasmic vacuolations at PND21. It has been reported that early-phase apoptosis is characterized by the indentation of the nuclear membrane. In the late phase, the chromatin becomes condensed and segregated along the 
margin of the nuclear membrane, and the cytoplasm shows densely packed organelles, including mitochondria and electron-dense vesicles [22]. Similar ultrastructural changes were observed in rat liver exposed to CYP [23]. Abnormal mitochondria were also noticed in the neurons of the CYPtreated group. The mitochondrial changes detected in this study may be an early sign of apoptosis and a mechanism by which the cell adapts to an unfavorable environment, such as free radicals [24].

Sharma et al. [17] explained these changes by oxidative stress as cypermethrin generates the ROS through lipid peroxidation and inhibits the antioxidant enzymes. The ROS attacks the neurons, disturbs the mitochondrial function, destructs the cellular macromolecules as (lipids and proteins), and finally causes the apoptosis [25]. The nerve fibers appeared immature and unmyelinated, the immaturity of nerve fibers indicated the delayed medullary development and loss of myelin sheath (produced by the wrapping oligodendrocyte cell) which is the early sign of neurodegeneration as reported by Alizadeh et al. [26]. This had been confirmed by the MAP-2 which had been significantly decreased in the cypermethrin treated medulla as compared to the control group. Also, immunohistochemically the medullary injury in the cypermethrin group had been confirmed by the significant decrease of MBP and Olig2. This is in agreement with Matesic and Lin [27] who stated that MAP-2 is an early indicator of neurodegeneration. The dysfunction of MAP-2 results in the impairment of axonal transport with the destruction of the microtubular structure, eventually causing apoptosis of neurons [28].

The increment of astrocyte number was confirmed by an increase in GFAP expression. Also, Betancourt et al. [29] observed increased GFAP mRNA levels in the forebrain on PND7 following oral exposure to organophosphorus insecticide. In response to neuronal degeneration and loss, the astrocytes increased in an attempt to limit the oxidative damage and inflammation [30, 31]. Mohamed and Eltony [32] revealed that the advanced astrogliosis might be a protective morphological change.

Prevention against oxidative stress can be obtained by the administration of chemical entities, either as individual drugs or as naturally occurring dietary constituents. In this respect, it was noticed that the concomitant administration of melatonin with cypermethrin showed the preservation of normal histomorphology. Similarly, Rao and Purohit [33] demonstrated that melatonin ameliorated the pathologi- cal changes in the medulla oblongata of the rat brain from mercury-induced toxicity. This is due to the potential neuroprotective effect of melatonin against apoptosis and oxidative stress in the brainstem of rats [34]. Moreover, melatonin is known to readily cross the blood-brain barrier [35] and maintain a healthy neuronal organization [36].

Our study revealed that co-treatment with melatonin upregulates MAP-2 expression in the medulla oblongata. The same results were reported by Meléndez et al. [37] who mentioned that this effect is due to an increase in the polymerization state of tubulin.

In this study, co-administration of melatonin significantly ameliorated the reduction in MBP and Olig2 immunoreactive structures in the medulla oblongata. This result suggested that melatonin protects against demyelination due to CYP-induced toxicity. Ghareghani et al. [38] explained that melatonin stimulated oligodendrogenesis, suggesting that oligodendrocytes are forced to use an alternative pathway to synthesize fatty acids for remyelination.

Co-administration of melatonin to CYP-treated rats lowered GFAP reactivity. Baydas et al. [39] said that melatonin likely inhibits astrocyte reactivity by potentiating the antioxidative defense system of these cells.

In conclusion, our findings showed that melatonin could have a potential neuroprotective effect against Cypermethrin-induced developmental toxicity in the medulla oblongata of rats. It attenuated the decrement of MAP-2, MBP, and Olig2 immuno-staining. Moreover, melatonin modulated the glial response. However, further experimental studies are needed to draw a definite conclusion in this regard.

\section{ORCID}

Marwa A. Al-Gholam:

https://orcid.org/0000-0002-1948-184X

Noha M. Issa: https://orcid.org/0000-0003-0420-7394

\section{Author Contributions}

Conceptualization: MAAG. Data acquisition: MAAG. Data analysis or interpretation: MAAG. Drafting of the manuscript: MAAG, NMI. Critical revision of the manuscript: MAAG, NMI. Approval of the final version of the manuscript: all authors. 


\section{Conflicts of Interest}

No potential conflict of interest relevant to this article was reported.

\section{References}

1. Sankar P, Telang AG, Manimaran A. Effect of piperine on cypermethrin-induced oxidative damage in rats. J Vet Sci Technol 2011;2:105.

2. Sankar P, Telang AG, Manimaran A. Curcumin protects against cypermethrin-induced genotoxicity in rats. Environ Toxicol Pharmacol 2010;30:289-91.

3. Hussien HM, Abdou HM, Yousef MI. Cypermethrin induced damage in genomic DNA and histopathological changes in brain and haematotoxicity in rats: the protective effect of sesame oil. Brain Res Bull 2013;92:76-83.

4. Huang F, Liu Q, Xie S, Xu J, Huang B, Wu Y, Xia D. Cypermethrin induces macrophages death through cell cycle arrest and oxidative stress-mediated JNK/ERK signaling regulated apoptosis. Int J Mol Sci 2016;17:885.

5. Ferrari CKB. Free radicals, lipid peroxidation and antioxidants in apoptosis: implications in cancer, cardiovascular and neurological diseases. Biologia 2000;55:581-90.

6. Sánchez A, Calpena AC, Clares B. Evaluating the oxidative stress in inflammation: role of melatonin. Int J Mol Sci 2015;16:16981-7004.

7. Wongprayoon P, Govitrapong P. Melatonin attenuates methamphetamine-induced neuroinflammation through the melatonin receptor in the SH-SY5Y cell line. Neurotoxicology 2015;50:122-30.

8. Kazemi M, Shokri S, Ganjkhani M, Ali R, Iraj JA. Modulation of axonal sprouting along rostro-caudal axis of dorsal hippocampus and no neuronal survival in parahippocampal cortices by long-term post-lesion melatonin administration in lithiumpilocarpine model of temporal lobe epilepsy. Anat Cell Biol 2016;49:21-33.

9. Selevan SG, Kimmel CA, Mendola P. Identifying critical windows of exposure for children's health. Environ Health Perspect 2000;108 Suppl 3:451-5.

10. Sayim F, Yavasoglu NÜK, Uyanikgil Y, Aktug H, Yavasoglu A, Turgut M. Neurotoxic effects of cypermethrin in wistar rats: a haematological, biochemical and histopathological study. J Health Sci 2005;51:300-307.

11. Issa NM, Al-Gholam MA. The effect of N-acetylcysteine on the sensory retina of male albino rats exposed prenatally to cypermethrin. Folia Morphol (Warsz) 2020 Apr 17 [Epub]. https://doi.org/10.5603/FM.a2020.0043.

12. Assayed ME, Khalaf AA, Salem HA. Protective effects of garlic extract and vitamin $\mathrm{C}$ against in vivo cypermethrin-induced cytogenetic damage in rat bone-marrow. Mutat Res 2010;702:17.

13. Tain YL, Huang LT, Lin IC, Lau YT, Lin CY. Melatonin pre- vents hypertension and increased asymmetric dimethylarginine in young spontaneous hypertensive rats. J Pineal Res 2010;49:390-8.

14. Huang C, Li X. Maternal cypermethrin exposure during the perinatal period impairs testicular development in C57BL male offspring. PLoS One 2014;9:e96781.

15. Luo J, Chen G, Wei L, Qian H, Lai X, Wang D, Lv J, Yu X. Severe diffuse axon injury in chronic alcoholic rat medulla oblongata following a concussion blow. Alcohol Alcohol 2014;49:2317.

16. Suvarna Kim, Layton CW, Bancroft JD. Bancroft's theory and practice of histological techniques. 7th ed. Oxford: Churchill Livingstone, 2013.

17. Sharma P, Firdous S, Singh R. Neurotoxic effect of cypermethrin and protective role of resveratrol in Wistar rats. Int J Nutr Pharmacol Neurol Dis 2014;4:104-11.

18. Elser BA, Kayali K, Dhakal R, O'Hare B, Wang K, Lehmler HJ, Stevens HE. Combined maternal exposure to cypermethrin and stress affect embryonic brain and placental outcomes in mice. Toxicol Sci 2020;175:182-96.

19. Dallegrave A, Pizzolato TM, Barreto F, Bica VC, Eljarrat E, Barceló D. Residue of insecticides in foodstuff and dietary exposure assessment of Brazilian citizens. Food Chem Toxicol 2018;115:329-35.

20. Tarocco A, Caroccia N, Morciano G, Wieckowski MR, Ancora G, Garani G, Pinton P. Melatonin as a master regulator of cell death and inflammation: molecular mechanisms and clinical implications for newborn care. Cell Death Dis 2019;10:317.

21. Grewal KK, Sandhu GS, Kaur R, Brar RS, Sandhu HS. Toxic impacts of cypermethrin on behavior and histology of certain tissues of albino rats. Toxicol Int 2010;17:94-8.

22. Tiso M, Gangemi R, Bargellesi Severi A, Pizzolitto S, Fabbi M, Risso A. Spontaneous apoptosis in human thymocytes. Am J Pathol 1995;147:434-44.

23. Abdul-Hamid M, Moustafa N, Abd Alla Asran AEM, Mowafy L. Cypermethrin-induced histopathological, ultrastructural and biochemical changes in liver of albino rats: the protective role of propolis and curcumin. Beni-Suef Univ J Basic Appl Sci 2017;6:160-73.

24. Wakabayashi T. Megamitochondria formation - physiology and pathology. J Cell Mol Med 2002;6:497-538.

25. Guo C, Sun L, Chen X, Zhang D. Oxidative stress, mitochondrial damage and neurodegenerative diseases. Neural Regen Res 2013;8:2003-14.

26. Alizadeh A, Dyck SM, Karimi-Abdolrezaee S. Myelin damage and repair in pathologic CNS: challenges and prospects. Front Mol Neurosci 2015;8:35.

27. Matesic DF, Lin RC. Microtubule-associated protein 2 as an early indicator of ischemia-induced neurodegeneration in the gerbil forebrain. J Neurochem 1994;63:1012-20.

28. Guo Y, Gong HS, Zhang J, Xie WL, Tian C, Chen C, Shi Q, Wang SB, Xu Y, Zhang BY, Dong XP. Remarkable reduction of MAP2 in the brains of scrapie-infected rodents and human prion disease possibly correlated with the increase of calpain. 
PLoS One 2012;7:e30163.

29. Betancourt AM, Burgess SC, Carr RL. Effect of developmental exposure to chlorpyrifos on the expression of neurotrophin growth factors and cell-specific markers in neonatal rat brain. Toxicol Sci 2006;92:500-6.

30. Chen X, Guo C, Kong J. Oxidative stress in neurodegenerative diseases. Neural Regen Res 2012;7:376-85.

31. Oksanen M, Lehtonen S, Jaronen M, Goldsteins G, Hämäläinen $\mathrm{RH}$, Koistinaho J. Astrocyte alterations in neurodegenerative pathologies and their modeling in human induced pluripotent stem cell platforms. Cell Mol Life Sci 2019;76:2739-60.

32. Mohamed HK, Eltony SA. Effect of acute pentylenetetrazol injection induced epileptic seizures on rat dentate gyrus at different postnatal ages. Anat Cell Biol 2020;53:84-94.

33. Rao MV, Purohit AR. Neuroprotection by melatonin on mercury induced toxicity in the rat brain. Pharmacol Pharm 2011;2:375-85.

34. Motallebzadeh E, Tameh AA, Zavareh SAT, Farhood B, Aliasgharzedeh A, Mohseni M. Neuroprotective effect of melatonin on radiation-induced oxidative stress and apoptosis in the brainstem of rats. J Cell Physiol 2020 Apr 23 [Epub]. https://doi. org/10.1002/jcp.29722.

35. Cheung RT. The utility of melatonin in reducing cerebral damage resulting from ischemia and reperfusion. J Pineal Res 2003;34:153-60.

36. Cohen-Mansfield J, Garfinkel D, Lipson S. Melatonin for treatment of sundowning in elderly persons with dementia - a preliminary study. Arch Gerontol Geriatr 2000;31:65-76.

37. Meléndez J, Maldonado V, Ortega A. Effect of melatonin on beta-tubulin and MAP2 expression in NIE-115 cells. Neurochem Res 1996;21:653-8.

38. Ghareghani M, Scavo L, Jand Y, Farhadi N, Sadeghi H, Ghanbari A, Mondello S, Arnoult D, Gharaghani S, Zibara K. Melatonin therapy modulates cerebral metabolism and enhances remyelination by increasing PDK4 in a mouse model of multiple sclerosis. Front Pharmacol 2019;10:147.

39. Baydas G, Reiter RJ, Yasar A, Tuzcu M, Akdemir I, Nedzvetskii VS. Melatonin reduces glial reactivity in the hippocampus, cortex, and cerebellum of streptozotocin-induced diabetic rats. Free Radic Biol Med 2003;35:797-804. 